\title{
On Hexagonal Structures in Higher Dimensional Theories
}

\author{
Adil Belhaj ${ }^{1 *}$ Luis J. Boya ${ }^{2 \dagger}$, Antonio Segui ${ }^{2 \ddagger}$ \\ ${ }^{1}$ Centre of Physics and Mathematics, CPM-CNESTEN, Rabat, Morocco \\ ${ }^{2}$ Departamento de Física Teórica, Universidad de Zaragoza, E-50009-Zaragoza, Spain
}

November 12, 2018

\begin{abstract}
We analyze the geometrical background under which many Lie groups relevant to particle physics are endowed with a (possibly multiple) hexagonal structure. There are several groups appearing, either as special holonomy groups on the compactification process from higher dimensions, or as dynamical string gauge groups; this includes groups like $\mathrm{SU}(2), \mathrm{SU}(3), G_{2}, \operatorname{Spin}(7), \mathrm{O}(8)$ as well as $E_{8}$ and $\mathrm{O}(32)$. We emphasize also the relation of these hexagonal structures with the octonion division algebra, as we expect as well eventually some role for octonions in the interpretation of symmetries in High Energy Physics.
\end{abstract}

KeyWords: Lie algebras, Strings, M and F-theories.

\footnotetext{
*belhaj@unizar.es

†luis.boya@gmail.com

łsegui@unizar.es
} 


\section{Introduction}

Symmetry is an essential ingredient of almost all physical theories. In particular, invariance under certain Lie groups characterizes e.g. Minkowski space and most of interactions are regulated also by certain gauge groups, like $\mathrm{SU}(3)$ (color), $\mathrm{SU}(2)$ and $\mathrm{U}(1)$ for the three forces of particle physics.

In this paper we remark the important rôle placed by hexagonal root structures in higher dimensional theories, all related to superstrings. This comes about because several compactification groups show in their root systems a hexagonal pattern. For such a group $G$, we have a relation between the dimension and the rank which reads

$$
\operatorname{Dim} G=\operatorname{rank} G+6 \times m,
$$

where $m$ is an integer specified later on.

The symmetry group is already the Lie group itself $G$, but besides the Weyl discrete symmetry group of the Dynkin diagram stresses, in our case, the hexagonal symmetry. At the moment, we just want to state these peculiarities and symmetries, leaving for further work to grasp the true meaning of that coincidences in string theory and related models.

To motivate the study, we first consider the traditional superstring theory, living in 10 dimensions [1]. If we impose $\mathcal{N}=1$ supersymmetry in our realizable 4-dimensional world, we are forced to compactify the remaining $10-4=6$ dimensions in a Calabi-Yau threefold $\left(\mathrm{CY}_{3}\right)$. The holonomy group [2] of a general $\mathrm{CY}_{n}$ manifold is $\mathrm{SU}(n)$, and in our $n=3$ case it is reduced to $\mathrm{SU}(3)$. Its dimension is $\operatorname{dim} \mathrm{SU}(3)=8=2+6=$ rank two + two simple roots + 4 reflections. In the corresponding Lie algebra noted $A_{2}$, there are the two simple roots $\alpha_{1}$ and $\alpha_{2}$ of equal length, and at $120^{\circ}$ angle. Its Dynkin diagram is as follows

$A_{2}$ :

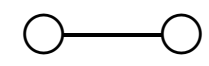

The total six nonzero roots come from these simple ones, with the sum and the opposed, to form the well known (single) hexagon. See Fig.-1-

The Weyl group of the $A_{2}(\equiv \mathrm{su}(3))$ Lie algebra is the symmetric group $S_{3}=D_{3}=Z_{3} \rtimes Z_{2}$ of order 6. It acts transitive and freely (= trivial stabilizer) on the hexagon.

This appearance of $\mathrm{SU}(3)$ in string theory is the more remarkable, as it is the third time this group appears in fundamental physics. We remember the original group SU(3) flavour of Gell-Mann (1962) [3], mixing the first three quarks flavors (up $u$, down $d$ and strange $s$ ). Later (1972), Gell-Mann and Fritzsch introduced also SU(3) as the gauge (color) group of the strong interactions[4], giving rise to QCD. At this point we only remark that the presence of 

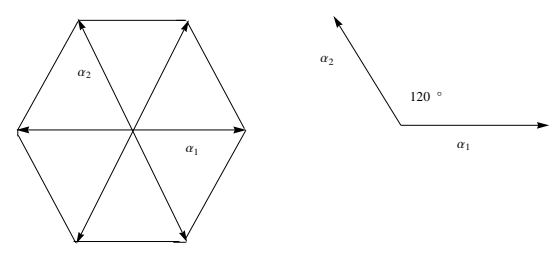

Figure 1: Root system of $A_{2}$.

the three SU(3) groups in microphysics seem to be completely unrelated with each other.

\section{Holonomy groups}

As we are to consider other holonomy groups, it is good to recall here briefly Berger classification of them, in this particular form, depending on the field of numbers and on some restrictions on the manifolds [2]. We shall follow the approach in [5]. A general (paracompact) $n$-dimensional manifold $\mathcal{M}$ admits always a Riemannian structure, whose generic holonomy group is the full orthogonal group $\mathrm{O}(n)$. If $\mathcal{M}$ is just orientable, the group becomes the connected rotation (sub)group $\mathrm{SO}(n)$; the condition for this is that the first Stiefel-Whitney class vanishes, $w_{1}(\mathcal{M})=0$. For complex Kähler manifolds of complex dimension $n$, the game is played by the unitary $\mathrm{U}(n)$ and the unitary unimodular (sub)group $\mathrm{SU}(n)$, and again the obstruction is measured by the first Chern class $c_{1}(\mathcal{M})$ of the (complex) tangent bundle. One completes the table with some quaternionic and octonionic groups. Here is the full classification of special holonomy groups [2, 5], as related to the four division number algebras $\mathbb{R}, \mathbb{C}, \mathbb{H}$ and $\mathrm{O}$ :

\begin{tabular}{lll} 
Number Field & General group & Unimodular Form \\
\hline $\mathbb{R}$ & $\mathrm{O}(n)$ & $\mathrm{SO}(n)$ (orientable, $\left.w_{1}=0\right)$ \\
$\mathbb{C}$ & $\mathrm{U}(n)$ (Kähler) & $\mathrm{SU}(n)\left(\right.$ Calabi-Yau, $\left.c_{1}=0\right)$ \\
$\mathbb{H}$ & $\mathrm{q}(n)$ & $\mathrm{Sq}(n)$ \\
$\mathrm{O}$ & $\operatorname{Spin}(7)$ in $8 d$ spaces & $G_{2}$ in $7 d$ spaces
\end{tabular}

For $\mathrm{q}(n)$ we mean the compact form of the non-simple group 


$$
\mathrm{q}(n)=\frac{\mathrm{q}(1) \times \mathrm{Sq}(n)}{Z_{2}}
$$

where $\mathrm{q}(1)=\mathrm{SU}(2)$ is the (multiplicative) group of unit quaternions $\mathbb{H}$, which form the three-sphere $S^{3} . \mathrm{Sq}(n)$ is the intersection of the unitary group with the real symplectic group, $\mathrm{Sq}(n):=\mathrm{U}(n) \cap \mathrm{Sp}(n)$. Today we have plenty of examples of manifolds with any of these special holonomy groups [6]. Notice all holonomy groups are compact, as subgroups of some real orthogonal group $\mathrm{O}(n)$.

As the octonion numbers $\mathrm{O}$ are not associative $\left(o_{1}\left(o_{2} o_{3}\right) \neq\left(o_{1} o_{2}\right) o_{3}\right.$ in general) only octonionic vector spaces could form up to three (octonionic) dimensions. In particular, the exceptional octonionic holonomy groups are only Spin(7), with a natural irreducible (real) representation of dimension $2^{(7-1) / 2}=8$, and the exceptional Lie group $G_{2}$, acting naturally in $\mathbb{R}^{7}$, i.e. in seven dimensions. The corresponding Dynkin diagrams are given by

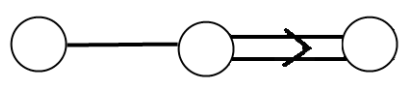

Spin (7)

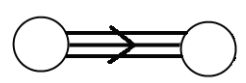

G2

Figure 2: Spin(7) and G2 Dynkin diagrams

We consider $G_{2}$ (with dimension 14) as the automorphism group of the octonions; if we write them as $o=v+\xi$, with $v$ the real part and $\xi$ a vector in $R^{7} ; v$ is unchanged under any automorphism, as Aut(real numbers)=Id. Therefore, as the norm $\mathcal{N}(o):=v^{2}+\xi \cdot \xi$ is real, so is also invariant; so $G_{2}$ acts naturally in the 6-sphere of unit imaginary octonions; one shows this action is transitive, so the stabilizer group (called little group in physics by Wigner) is of dimension $14-6=8$; it turns out to be $\mathrm{SU}(3)$. Analogously, the $O(7)$ spin representation $\Delta$ has dimension $\Delta=2^{(7-1) / 2}=8$ (and real type), and the natural action of $\operatorname{Spin}(7)$ on the 7 -sphere is also transitive, with stabilizer of dimension $7 \cdot 6 / 2-7=14$; indeed it is the group $G_{2}$ again (See [16]). If one imagines this 7-sphere as the set of all unit octonions, the imaginary ones collect themselves in the equator $\approx S^{6}$, and the mentioned stabilizer SU(3) acts in the whole ambient space $R^{6}$ via the $6=3+\overline{3}$ representations. This "octonionic" presentation of SU(3) may very well be, on the long run, the very reason of the presence so repeated of SU(3) in microphysics.

\section{Hexagonal structures in $\mathrm{M}$ and F-theories}

In the "second" superstring revolution (1995), Witten [7] showed how the five existing superstring theories, living in ten dimensions, were subsumed in a certain "M-Theory", working 
in eleven dimensions, and encompassing maximal supergravity (32 supercharges).

As Townsend et al. stated in [8] the corresponding seven-dimensional $(7=11-4)$ compactifying manifold has most likely $G_{2}$ holonomy and this group also exhibits a hexagonal root structure with rank 2 and dimension 14 :

$$
14=2+6 \times 2
$$

So we have two hexagons of unequal side length, with primary unequal roots at angle $60^{\circ}+$ $90^{\circ}=150^{\circ}$. Two unequal roots each generates an hexagon (we refrain to copy the star-shaped figure; see $[9,10])$. The Weyl group of the $G_{2}$ diagram is the dihedral group

$$
D_{6}=Z_{6} \rtimes Z_{2}
$$

of order 12 which acts transitively (stabilizer $Z_{2}$ ) on the two hexagons independently.

To see the nontriviality of our approach, notice e.g. that for $B_{2}=C_{2}$ does not work: $10=2+2 \times 4:$ no hexagons here! The figure is that of a square with diagonals. The two fundamental irreps of $G_{2}$, associated with its diagram are 7 and 14-dimensional (the first acts in unit imaginary octonions, as said, and the other is the adjoint). We wish to specify the action of these groups on spheres.

The group SU(3) has the complex representation 3, and SU(3) being unitary it leaves the 5-sphere $S^{5} \subset R^{6}=C^{3}$ invariant; indeed, that action is again transitive, with little group $\mathrm{SU}(2): \mathrm{SU}(3) / \mathrm{SU}(2) \approx S^{5}$, or as an exact suite:

$$
\mathrm{SU}(2) \rightarrow \mathrm{SU}(3) \rightarrow S^{5}
$$

On the other hand, as we explained above, $G_{2} / S U(3) \approx S^{6}$ also, so we have another exact sequence

$$
\mathrm{SU}(3) \rightarrow G_{2} \rightarrow S^{6}
$$

The IIB superstring theory did not fit very well in the original M-Theory, so C. Vafa postulated [11], (1996) a further extension to 12 dimensions. Today this is an ample field of research, still growing, [12]; for more work see e.g. the recent F-theory paper in relation to GUT [13]. Working with F-theory, we need a $12-4=8$ dimensional compactifying manifold. Candidate groups are $\mathrm{O}(8), \mathrm{SO}(8)$, $\operatorname{Spin}(7)$ and $\mathrm{SU}(4)$, all with a natural real 8$\operatorname{dim}$ representation. Spin $(7)$ is the natural generalization of $G_{2}$, as it can be understood also as an octonionic group. It has a natural spinor $8=2^{(7-1) / 2}$-dimensional real representation, therefore it acts naturally in the seven sphere of all unit octonions. It is also one of the holonomy groups in Berger's list, see above. The $\mathrm{O}(7)$ group has dimension $7 \cdot 6 / 2=21$, and 
rank three: it is $B_{3} \neq C_{3}$ in Cartan's list; hence, the number of nonzero roots is

$$
(\operatorname{Dim} G-\operatorname{rank} G)=(21-3)=18=6 \cdot 3,
$$

so it does have an hexagonal structure.

This is seen also from the Dynkin diagram $B_{3}$ : interpreted as the Weyl symmetry group. This Weyl group $W\left(B_{3}\right)=Z_{2}^{3} \rtimes S_{3}$, of order 48 , has three involutive generators, say $a, b, c$ with relations

$$
a^{2}=b^{2}=c^{2}=(a b)^{3}=(b c)^{4}=(a c)^{2}=e
$$

The biplane $\langle a, b\rangle$ generates a hexagon as before (group $\mathrm{SU}(3)$ ). The biplane $\langle b, c\rangle$ generates a square (with diagonal and sides), with symmetry group $D_{4}=Z_{4} \rtimes Z_{2}$, and the biplane $\langle a, c\rangle$ with orthogonal fundamental roots generates an unequal cross, with group $V=Z_{2} \times Z_{2}$. The counting of nonzero roots is

$$
18=21-3=6(\text { hexagon })+8(\text { diagonalized square })+4(\text { unequal cross })
$$

Thus we see here again a hexagon emerging.

So the three standard compactifying groups show hexagons: $\operatorname{Spin}(7), G_{2}$ and SU(3), and the three are also related to the octonions. To call attention to this fact is the main purpose of this paper.

As $\operatorname{Spin}(7)$ lies naturally in $\mathrm{SO}(8)$, which acts also naturally in $S^{7}$, it is tempting to amplify the scheme including $\mathrm{SO}(8)$. The Dynkin diagram for $D_{4}$ is very special (Figure 3 ).

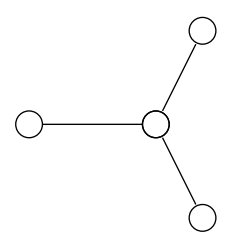

Figure 3: $D_{4}$ Dynkin diagram

Notice that the $D_{4}$ Dynkin Diagram is the only diagram with outer symmetry beyond $Z_{2}$, as in the $A$ and $D$ series and $E_{6}$. The three external roots correspond to the three $\operatorname{dim} 8$ 
irreps: vector and the two spinor $\Delta_{L, R}$, permuted by triality, as there is a large group of outer automorphisms:

$$
\operatorname{Out}(\operatorname{Spin}(8))=S_{3}
$$

We believe this triality would play a rôle in understanding the hexagon replicas (and hopefully other features of the physics), but at the moment we do not elaborate.

We just comment an aspect of this diagram. The central root (which corresponds to the adjoint irrep, of dimension 28) with any of the 3 outer ones generates three hexagons, as each has the structure of $A_{2}$. The three outside roots by themselves generate (as involutions) $\left(Z_{2}\right)^{3}$, as they are disconnected and hence orthogonals. In general, the hexagon structure arises because the numerical coincidence $\operatorname{Dim} G=\operatorname{rank}+6 \times m$, where $m$ is now 4 :

$$
\operatorname{Dim} \mathrm{O}(8)=8 \times 7 / 2=28=4(\text { rank })+6 \times 4
$$

Now the nine mentioned groups can be assembled together after the inclusions

$$
\begin{array}{cccccccccc}
\text { Group: } & \mathrm{SU}(2) & \subset & \mathrm{SU}(3) & \subset & \mathrm{G}_{2} & \subset & \operatorname{Spin}(7) & \subset & \mathrm{SO}(8) \\
\operatorname{Dim} & 3 & & 8 & & 14 & & 21 & & 28
\end{array}
$$

Together with the natural ones

$$
\begin{aligned}
& \mathrm{SU}(2) \subset \mathrm{SU}(2)^{2}=\mathrm{Spin}(4) \rightarrow \mathrm{SO}(4) \\
& \mathrm{Spin}(7) \supset \mathrm{Spin}(5) \\
& \mathrm{SU}(4) \subset \mathrm{SO}(8) \supset \mathrm{SU}(7)
\end{aligned}
$$

¿From the Dynkin diagram for $D_{4}$, one gets by truncation (removing a node) and folding (collapsing under external automorphisms) all the groups above; it is a standard exercise in Lie groups. Notice also the equivalences (or Cartan identities)

$$
\begin{aligned}
A_{1}+A_{1} & =D_{2}(\operatorname{group} \mathrm{O}(4)), \text { or } \operatorname{Spin}(4)=(\operatorname{Spin}(3))^{2} \\
A_{3} & =D_{3}, \text { or } \mathrm{SU}(4)=\operatorname{Spin}(6) .
\end{aligned}
$$

By folding we get $B_{2}=\operatorname{Spin}(5)=\operatorname{Sp}(2)=C_{2}$.

Now we see the " 6 " number for all holonomy groups. The following Table sums up the situation: 


\begin{tabular}{lll} 
Holonomy group & Dimension & Decomposition \\
\hline $\mathrm{O}(8)$ & 28 & $4+6 \times 4$ \\
$\mathrm{Spin}(7)$ & 21 & $3+6 \times 3$ \\
$\mathrm{G}_{2}$ & 14 & $2+6 \times 2$ \\
$\mathrm{SU}(4)$ & 15 & $3+6 \times 2$ \\
$\mathrm{SU}(3)$ & 8 & $2+6 \times 1$,
\end{tabular}

We see that all include hexagons.

\section{Discussion}

It seems remarkable to us that even the large groups used as gauge groups in string theory, and related models, can group the nonzero roots in some hexagons. $E_{8}$ appears as itself in M-theory [14], and as squared $E_{8} \times E_{8}$ in one of the heterotic strings. But, in dimensions 248 and $\operatorname{rank}(8)$ we have again nonzero roots grouped in hexagons: in dealing with simply laced groups, there is an hexagon for each $A_{2}$ bond for example, in $E_{8}$

$$
\operatorname{Dim} E_{8}=248=8(\text { rank })+6 \times 40 .
$$

As for the other heterotic string group, $\mathrm{SO}(32)$, it is also a $D$ series groups, which is simply laced one, and it should include hexagons. Indeed, we have

$$
\text { Dim SO(32) }=32 \times 31 / 2=496=\text { third perfect number }=16(\text { rank })+6 \times 80 \text {. }
$$

We realize it is hard to imagine any role for these numerous hexagons, but the numbers are there. Also, at face value we do not see any rôle for the octonions.

We cannot skip the appearance of hexagonal structures in other domains of physics. We add this just to reinforce our point of view; in particular hexagons, dual triangles and squares tessellate the plane $R^{2}$. For another example, in solid state physics, the structure of graphene [15] (or laminar graphite) has been shown a hexagonal structure, linked to the benzene hexagon. One also expects some hexagonal structures to arise also in valence-four elements, as carbon; in particular, silicium(Si) and germanium(Ge) are expected to have hexagonal structures also.

Acknowledgments: AB would like to thank the grant A/031268/10 and the Department of Theoretical Physics at Zaragoza University for very kind hospitality. He is also very grateful 
to M. Asorey for scientific help. This work has been also supported by the grants FPA200909638 (CICYT), 2011-E24/2 (DGHD-DGA) and A/031268/10.

\section{References}

[1] M. Green, J. Schwarz and E. Witten, Superstring Theory, 2 Vols., Cambridge U.P.1987.

[2] M. Berger, Bull. Soc. Math. France 83 (1953) 279-330.

[3] M. Gell-Mann, Y. Ne'eman, The Eightfold Way, Benjamin, New York (1964).

[4] M. Gell-Mann, H. Fritsch and H. Leutwiller, Phys. Lett. 47B (1973) 365.

[5] L. J. Boya, Special Holonomy Manifolds in Physics, R. Acad. Ciencias de Zaragoza 29,(2006) 37-47, math-ph/0612002

[6] D. Joyce, Compact Manifolds with Special Holonomy, Oxford U.P. 2000.

[7] E. Witten, String Theory Dynamics In Various Dimensions, Nucl.Phys.B443(1995)85-126, hep-th/9503124.

[8] N. Papadopoulos and P. K.Townsend, Phys. Lett B380, (1996), 273.

[9] N. Jacobson Lie, Algebras, Dover (1962).

[10] H. Weyl, Theory of Groups and Quantum Mechanics, Dover 1963.

[11] C. Vafa, Evidence for F-Theory, Nucl.Phys.B469(1996)403-418, hep-th/9602022.

[12] L.J. Boya, Arguments for F-theory, Mod.Phys.Lett. A21 (2006) 287-304, hep-th/0512047.

[13] C. Beasley, J. J. Heckman, C. Vafa, GUTs and Exceptional Branes in F-theory - I, JHEP 0901:058,2009, arXiv:0802.3391.

[14] E. Diaconescu, D. S. Freed, G. Moore, The M-theory 3-form and E gauge theory, hep-th/0312069.

[15] A.K. Geim, K.S. Novoselov, The rise of graphene, Nature Materials 6, (2007) 183.

[16] J.F. Adams, Lectures on Exceptional Lie Groups. University of Chicago Press, 1996. 